\title{
Review Article \\ Meta-Analysis of the Association between Vitiligo and Human Leukocyte Antigen-A
}

\author{
Zhangjun Li, ${ }^{1}$ Jianwen Ren, ${ }^{1}$ Xinwu Niu, ${ }^{1}$ Qingqiang Xu, ${ }^{2}$ Xiaopeng Wang, \\ Yale Liu, ${ }^{1}$ and Shengxiang Xiao ${ }^{1}$ \\ ${ }^{1}$ Department of Dermatology, Second Affiliated Hospital of Xian Jiaotong University, \\ Xian 710004, China \\ ${ }^{2}$ Department of Dermatology, Traditional Chinese Medicine Hospital of Shaanxi Province, \\ Xian 710003, China \\ Correspondence should be addressed to Shengxiang Xiao; xiao_sx@163.com
}

Received 24 May 2016; Revised 22 July 2016; Accepted 4 August 2016

Academic Editor: Davinder Parsad

Copyright (C) 2016 Zhangjun Li et al. This is an open access article distributed under the Creative Commons Attribution License, which permits unrestricted use, distribution, and reproduction in any medium, provided the original work is properly cited.

Objective. The objective of this study was to systematically evaluate the association between vitiligo and human leukocyte antigen(HLA-) A. Methods. PubMed, Embase, Web of Science, Chinese National Knowledge Infrastructure, and reference lists were searched for relevant original articles. Results. Nineteen case-control studies comprising 3042 patients and 5614 controls were included, in which 33 HLA-A alleles were reported. Overall, three alleles (HLA-A*02, A*33, and Aw 31 ) were significantly associated with increased risk of vitiligo, two (HLA-A 09 and Aw $\left.^{*} 19\right)$ were associated with decreased risk, and the remaining 28 were unassociated. Twelve alleles, seven alleles, and 19 alleles were common to three ethnicities, both types of vitiligo, and both typing methods, respectively. In the subgroup analysis by ethnicity and typing methods, the association of six alleles and five alleles was inconsistent in three populations and both typing methods, respectively. In the subgroup analysis by clinical type, the association of all seven alleles was consistent in both types of vitiligo. Conclusion. The meta-analysis suggests that HLA- $\mathrm{A}^{*} 02, \mathrm{~A}^{*} 33$, and $\mathrm{Aw}^{*} 31$ are associated with increased risk of vitiligo, while HLA-A* 09 and $\mathrm{Aw}^{*} 19$ are associated with decreased risk of vitiligo. The association of some alleles varies in terms of ethnicity and typing methods.

\section{Introduction}

Vitiligo is an acquired depigmentation disorder of the skin characterized by absence of functional melanocytes. It affects approximately $0.5-2 \%$ of the world's population and impairs the patients' quality of life $[1,2]$. The exact pathogenesis of vitiligo remains unknown; however, many potential theories have been proposed, including autoimmune, neural, genetic, melanocytorrhagy, and reactive oxygen species model hypotheses [3]. Among these, the autoimmune hypothesis is currently most widely accepted because of the frequent occurrence of other concomitant autoimmune diseases $[4,5]$ and the presence of circulating autoantibodies against pigment cells $[6,7]$. Several genetic epidemiological studies have also demonstrated that genetic factors play an important role in the pathogenesis of vitiligo $[8,9]$.
The inherited nature of vitiligo and its frequent association with autoimmune diseases have prompted numerous studies on the association of vitiligo with human leukocyte antigens (HLAs), especially with HLA-A [10-26]. However, the results of these studies are controversial due to distinct ethnic populations, small sample sizes, and different research methods. With the development of molecular biology, genome-wide association studies have been successful in identifying susceptibility loci of vitiligo. Some authors have found that vitiligo is associated with HLA-A locus in Caucasians and the Japanese [27, 28]. A previous metaanalysis suggested that HLA-A2 was significantly associated with vitiligo [29], but the quality and strength of evidence were limited by the number of included studies. Moreover, newly published studies showed no association between vitiligo and HLA-A2 [10, 11, 13, 14]. 


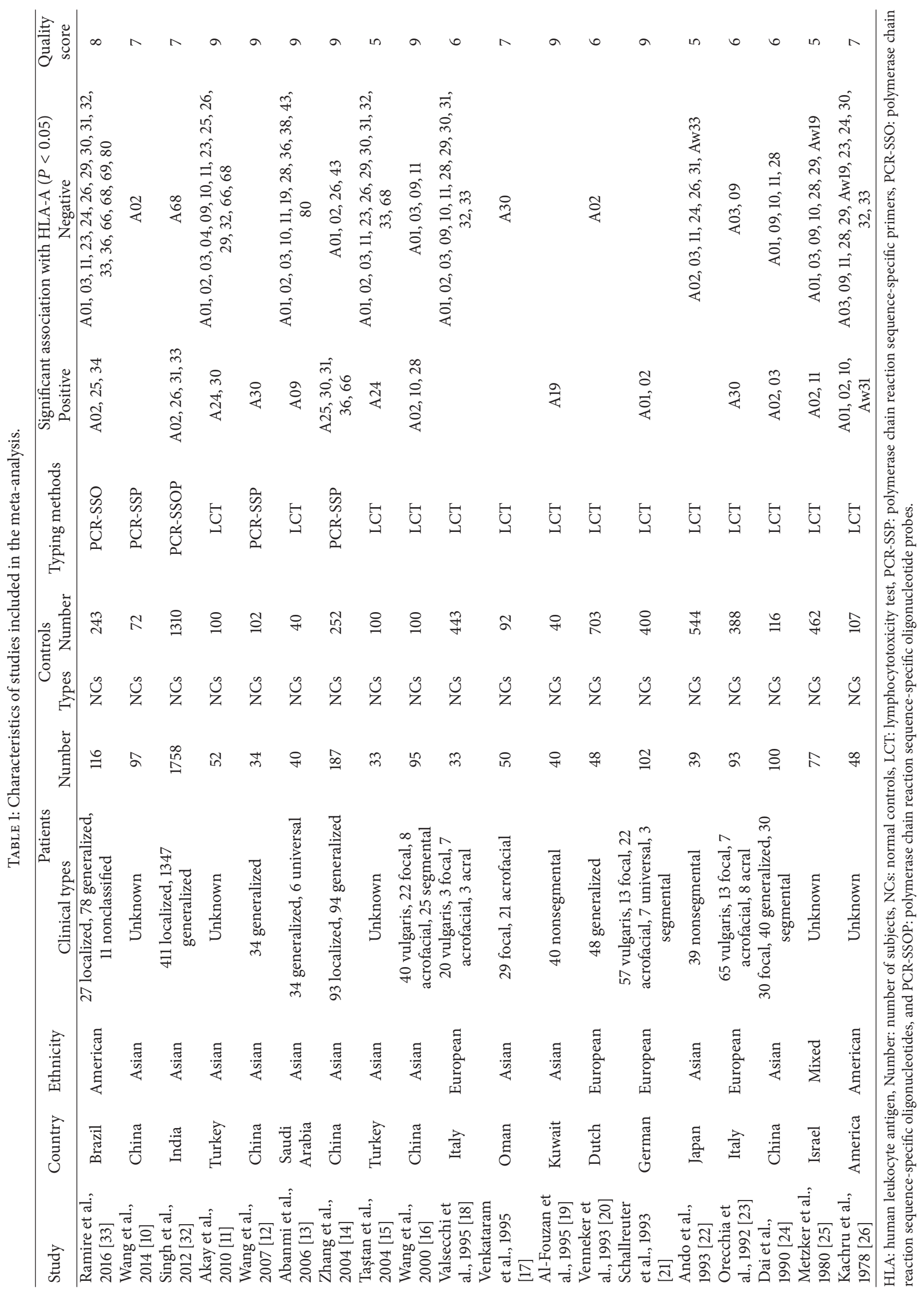


TABLE 2: Association between vitiligo and 33 human leukocyte antigen- (HLA-) A alleles.

\begin{tabular}{|c|c|c|c|c|}
\hline Allele & Patients\% $(n / N)$ & Controls $\%(n / N)$ & OR (95\% CI)/article number & $P$ \\
\hline $\mathrm{A}^{*} 01$ & $28.20(249 / 883)$ & $28.65(677 / 2363)$ & $0.95 \mathrm{r}(0.66,1.35) / 11$ & 0.761 \\
\hline $\mathrm{A}^{*} 02$ & $26.94(761 / 2825)$ & $34.25(1710 / 4992)$ & $1.52 \mathrm{r}(1.21,1.90) / 15$ & $<0.001$ \\
\hline $\mathrm{A}^{*} 03$ & $19.70(143 / 726)$ & $14.30(378 / 2643)$ & $1.45 \mathrm{r}(0.99,2.13) / 11$ & 0.056 \\
\hline $\mathrm{A}^{*} 04$ & $3.85(2 / 52)$ & $3.00(3 / 100)$ & $1.29(0.21,7.99) / 1$ & 0.782 \\
\hline$A^{*} 09$ & $20.07(108 / 538)$ & $26.25(461 / 1756)$ & $0.70(0.54,0.90) / 8$ & 0.005 \\
\hline $\mathrm{A}^{*} 10$ & $10.56(47 / 445)$ & $12.79(175 / 1368)$ & $1.11 \mathrm{r}(0.51,2.41) / 7$ & 0.786 \\
\hline$A^{*} 11$ & $15.80(100 / 633)$ & $14.41(325 / 2255)$ & $0.87(0.66,1.14) / 10$ & 0.306 \\
\hline$A^{*} 19$ & $22.50(18 / 80)$ & $43.75(35 / 80)$ & $0.19 \mathrm{r}(0.02,2.02) / 2$ & 0.170 \\
\hline$A^{*} 23$ & $5.47(11 / 201)$ & $7.22(32 / 443)$ & $0.76(0.38,1.53) / 3$ & 0.436 \\
\hline $\mathrm{A}^{*} 24$ & $21.67(52 / 240)$ & $40.53(400 / 987)$ & $0.89 \mathrm{r}(0.36,2.21) / 4$ & 0.800 \\
\hline$A^{*} 25$ & $11.83(42 / 355)$ & $5.71(34 / 595)$ & $1.29 \mathrm{r}(0.26,6.43) / 3$ & 0.756 \\
\hline$A^{*} 26$ & $8.05(176 / 2185)$ & $13.42(342 / 2549)$ & $0.79 \mathrm{r}(0.53,1.19) / 6$ & 0.265 \\
\hline$A^{*} 28$ & $7.89(31 / 393)$ & $8.60(109 / 1268)$ & $0.74(0.46,1.17) / 6$ & 0.192 \\
\hline$A^{*} 29$ & $6.13(22 / 359)$ & $7.90(115 / 1455)$ & $0.78(0.49,1.27) / 6$ & 0.320 \\
\hline$A^{*} 30$ & $12.37(74 / 598)$ & $6.92(119 / 1720)$ & $1.83 \mathrm{r}(0.88,3.81) / 8$ & 0.107 \\
\hline$A^{*} 31$ & $3.88(84 / 2166)$ & $6.54(189 / 2892)$ & $1.33 \mathrm{r}(0.61,2.90) / 6$ & 0.479 \\
\hline$A^{*} 32$ & $6.41(15 / 234)$ & $8.24(73 / 886)$ & $0.78(0.43,1.40) / 4$ & 0.400 \\
\hline$A^{*} 33$ & $22.16(430 / 1940)$ & $8.78(184 / 2096)$ & $2.23(1.84,2.70) / 4$ & $<0.001$ \\
\hline$A^{*} 34$ & $0.00(0 / 116)$ & $3.29(8 / 243)$ & $0.12(0.01,2.08) / 1$ & 0.145 \\
\hline$A^{*} 36$ & $0.58(2 / 343)$ & $2.24(12 / 535)$ & $0.43 \mathrm{r}(0.04,5.02) / 3$ & 0.501 \\
\hline$A^{*} 38$ & $0.00(0 / 40)$ & $2.50(1 / 40)$ & $0.33(0.01,8.22) / 1$ & 0.495 \\
\hline$A^{*} 43$ & $31.72(72 / 227)$ & $36.64(107 / 292)$ & $0.85(0.58,1.24) / 2$ & 0.394 \\
\hline$A^{*} 66$ & $2.82(10 / 355)$ & $7.73(46 / 595)$ & $0.58 \mathrm{r}(0.08,4.25) / 3$ & 0.595 \\
\hline$A^{*} 68$ & $14.45(283 / 1959)$ & $12.09(212 / 1753)$ & $1.12(0.93,1.36) / 4$ & 0.238 \\
\hline$A^{*} 69$ & $0.00(0 / 116)$ & $0.82(2 / 243)$ & $0.41(0.02,8.71) / 1$ & 0.571 \\
\hline $\mathrm{A}^{*} 80$ & $1.28(2 / 156)$ & $1.77(5 / 283)$ & $0.52(0.11,2.43) / 2$ & 0.405 \\
\hline $\mathrm{Aw}^{*} 19$ & $0.72(13 / 1806)$ & $6.35(90 / 1417)$ & $0.11(0.06,0.19) / 2$ & $<0.001$ \\
\hline $\mathrm{Aw}^{*} 23$ & $2.08(1 / 48)$ & $4.67(5 / 107)$ & $0.43(0.05,3.82) / 1$ & 0.452 \\
\hline $\mathrm{Aw}^{*} 24$ & $0.00(0 / 48)$ & $2.80(3 / 107)$ & $0.31(0.02,6.08) / 1$ & 0.439 \\
\hline $\mathrm{Aw}^{*} 30$ & $20.83(10 / 48)$ & $28.04(30 / 107)$ & $0.68(0.30,1.52) / 1$ & 0.345 \\
\hline $\mathrm{Aw}^{*} 31$ & $16.67(8 / 48)$ & $4.67(5 / 107)$ & $4.08(1.26,13.22) / 1$ & 0.019 \\
\hline $\mathrm{Aw}^{*} 32$ & $4.17(2 / 48)$ & $8.41(9 / 107)$ & $0.47(0.10,2.28) / 1$ & 0.351 \\
\hline $\mathrm{Aw}^{*} 33$ & $9.20(8 / 87)$ & $12.29(80 / 651)$ & $1.03(0.46,2.30) / 1$ & 0.944 \\
\hline
\end{tabular}

$N$ : total number of subjects, $n$ : positive number of subjects, OR: odds ratio, CI: confidence interval, article number: total number of the articles relevant to the association between vitiligo and HLA-A alleles, r: random effects model, and the others: fixed effects model.

Therefore, the objective of this meta-analysis was to systematically evaluate the association between vitiligo and HLA-A.

\section{Methods}

2.1. Search Strategy. Four electronic databases, PubMed, Embase, Web of Science, and Chinese National Knowledge Infrastructure (CNKI), were searched to screen all the casecontrol studies on the association between vitiligo and HLA, using free text and the Medical Subject Headings (MeSH) terms "vitiligo," "human leukocyte antigen," "HLA," "major histocompatibility complex," and "MHC." The search period was from the start of each database up to February 2016, and articles were published in either English or Chinese. Moreover, reference lists from the retrieved articles were checked manually for additional studies.
2.2. Criteria for Inclusion and Exclusion. Studies were included if they met the following criteria: (1) primary studies exploring the association between vitiligo and HLA-A; (2) case-control design; (3) studies with full-text articles; (4) studies presenting sufficient data for calculating odds ratios (ORs); and (5) serological and molecular methods used for HLA-A typing. Exclusion criteria were as follows: (1) no original research (reviews, abstracts, editorials, case reports, and nonresearch letters); (2) studies without control subjects; (3) incomplete raw data; and (4) duplicate articles or reused data.

2.3. Data Extraction and Quality Assessment. Two investigators (Zhangjun Li and Jianwen Ren) independently extracted data from all eligible studies. Any disagreements were resolved by discussion and consensus with a third investigator (Shengxiang Xiao). The following data were recorded: first 
TABLE 3: Association between vitiligo and human leukocyte antigen- (HLA-) A alleles in terms of ethnicity.

\begin{tabular}{|c|c|c|c|c|c|}
\hline Ethnicity & Allele & Patients $\%(n / N)$ & Controls $\%(n / N)$ & OR (95\% CI)/article number & $P$ \\
\hline \multirow{26}{*}{ Asian } & $\mathrm{A}^{*} 01$ & $32.54(165 / 507)$ & $34.04(241 / 708)$ & $0.91(0.66,1.26) / 6$ & 0.574 \\
\hline & $A^{*} 02$ & $21.45(515 / 2401)$ & $26.31(693 / 2634)$ & $1.24 \mathrm{r}(0.93,1.67) / 9$ & 0.147 \\
\hline & $\mathrm{A}^{*} 03$ & $16.71(60 / 359)$ & $5.00(50 / 1000)$ & $2.46 \mathrm{r}(1.11,5.45) / 6$ & 0.026 \\
\hline & $\mathrm{A}^{*} 04$ & $3.85(2 / 52)$ & $3.00(3 / 100)$ & $1.29(0.21,7.99) / 1$ & 0.782 \\
\hline & $\mathrm{A}^{*} 09$ & $18.82(54 / 287)$ & $24.72(88 / 356)$ & $0.60(0.40,0.89) / 4$ & 0.010 \\
\hline & $\mathrm{A}^{*} 10$ & $10.45(30 / 287)$ & $3.65(13 / 356)$ & $2.73(1.40,5.35) / 4$ & 0.003 \\
\hline & $\mathrm{A}^{*} 11$ & $22.84(82 / 359)$ & $20.10(201 / 1000)$ & $0.99(0.71,1.38) / 6$ & 0.960 \\
\hline & $\mathrm{A}^{*} 19$ & $22.50(18 / 80)$ & $43.75(35 / 80)$ & $0.19 \mathrm{r}(0.02,2.02) / 2$ & 0.170 \\
\hline & $A^{*} 23$ & $2.35(2 / 85)$ & $4.00(8 / 200)$ & $0.72(0.17,3.11) / 2$ & 0.662 \\
\hline & $\mathrm{A}^{*} 24$ & $25.00(31 / 124)$ & $48.12(358 / 744)$ & $0.79 \mathrm{r}(0.17,3.76) / 3$ & 0.767 \\
\hline & $\mathrm{A}^{*} 25$ & $17.57(42 / 239)$ & $7.10(25 / 352)$ & $2.65(1.56,4.51) / 2$ & $<0.001$ \\
\hline & $A^{*} 26$ & $8.07(167 / 2069)$ & $13.96(322 / 2306)$ & $0.76 \mathrm{r}(0.47,1.24) / 5$ & 0.274 \\
\hline & $A^{*} 28$ & $7.66(18 / 235)$ & $11.33(29 / 256)$ & $0.43 \mathrm{r}(0.08,2.20) / 3$ & 0.311 \\
\hline & $A^{*} 29$ & $2.35(2 / 85)$ & $5.00(10 / 200)$ & $0.45(0.10,2.08) / 2$ & 0.307 \\
\hline & $\mathrm{A}^{*} 30$ & $13.20(47 / 356)$ & $6.50(42 / 646)$ & $2.18 \mathrm{r}(0.85,5.61) / 5$ & 0.107 \\
\hline & $\mathrm{A}^{*} 31$ & $3.72(75 / 2017)$ & $6.53(144 / 2206)$ & $1.79 \mathrm{r}(0.55,5.85) / 4$ & 0.335 \\
\hline & $A^{*} 32$ & $8.24(7 / 85)$ & $9.00(18 / 200)$ & $0.90(0.36,2.23) / 2$ & 0.813 \\
\hline & $A^{*} 33$ & $23.56(422 / 1791)$ & $11.28(159 / 1410)$ & $2.32(1.90,2.83) / 2$ & $<0.001$ \\
\hline & $A^{*} 36$ & $0.89(2 / 227)$ & $2.74(8 / 292)$ & $0.61 \mathrm{r}(0.01,40.53) / 2$ & 0.818 \\
\hline & $A^{*} 38$ & $0.00(0 / 40)$ & $2.50(1 / 40)$ & $0.33(0.01,8.22) / 1$ & 0.495 \\
\hline & $A^{*} 43$ & $31.72(72 / 227)$ & $36.64(107 / 292)$ & $0.85(0.58,1.24) / 2$ & 0.394 \\
\hline & $A^{*} 66$ & $2.93(7 / 239)$ & $12.50(44 / 352)$ & $0.20(0.09,0.45) / 2$ & $<0.001$ \\
\hline & $A^{*} 68$ & $14.38(265 / 1843)$ & $12.58(190 / 1510)$ & $1.08(0.88,1.32) / 3$ & 0.476 \\
\hline & $\mathrm{A}^{*} 80$ & $5.00(2 / 40)$ & $10.00(4 / 40)$ & $0.47(0.08,2.75) / 1$ & 0.405 \\
\hline & $\mathrm{Aw}^{*} 19$ & $0.74(13 / 1758)$ & $6.79(89 / 1310)$ & $0.10(0.06,0.18) / 1$ & $<0.001$ \\
\hline & $\mathrm{Aw}^{*} 33$ & $12.82(5 / 39)$ & $13.79(75 / 544)$ & $0.92(0.35,2.43) / 1$ & 0.866 \\
\hline \multirow{12}{*}{ European } & $\mathrm{A}^{*} 01$ & $16.30(22 / 135)$ & $27.88(235 / 843)$ & $0.46(0.28,0.74) / 2$ & 0.002 \\
\hline & $\mathrm{A}^{*} 02$ & $62.84(115 / 183)$ & $41.01(757 / 1546)$ & $1.80(1.30,2.48) / 3$ & $<0.001$ \\
\hline & $\mathrm{A}^{*} 03$ & $25.40(32 / 126)$ & $20.58(171 / 831)$ & $1.23(0.79,1.92) / 2$ & 0.359 \\
\hline & $\mathrm{A}^{*} 09$ & $20.63(26 / 126)$ & $24.43(203 / 831)$ & $0.79(0.50,1.26) / 2$ & 0.321 \\
\hline & $\mathrm{A}^{*} 10$ & $9.09(3 / 33)$ & $13.09(58 / 443)$ & $0.66(0.20,2.25) / 1$ & 0.510 \\
\hline & $\mathrm{A}^{*} 11$ & $15.15(5 / 33)$ & $9.71(43 / 443)$ & $1.66(0.61,4.53) / 1$ & 0.321 \\
\hline & $A^{*} 28$ & $9.09(3 / 33)$ & $5.42(24 / 443)$ & $1.75(0.50,6.13) / 1$ & 0.385 \\
\hline & $A^{*} 29$ & $9.09(3 / 33)$ & $7.45(33 / 443)$ & $1.24(0.36,4.29) / 1$ & 0.731 \\
\hline & $A^{*} 30$ & $9.52(12 / 126)$ & $4.81(40 / 831)$ & $1.70 \mathrm{r}(0.16,18.02) / 2$ & 0.660 \\
\hline & $\mathrm{A}^{*} 31$ & $9.09(3 / 33)$ & $4.97(22 / 443)$ & $1.91(0.54,6.76) / 1$ & 0.313 \\
\hline & $A^{*} 32$ & $12.12(4 / 33)$ & $7.90(35 / 443)$ & $1.61(0.53,4.83) / 1$ & 0.398 \\
\hline & $\mathrm{A}^{*} 33$ & $6.06(2 / 33)$ & $2.26(10 / 443)$ & $2.79(0.59,13.31) / 1$ & 0.197 \\
\hline \multirow{12}{*}{ American } & $\mathrm{A}^{*} 01$ & $24.39(40 / 164)$ & $16.29(57 / 350)$ & $1.91 \mathrm{r}(0.66,5.53) / 2$ & 0.230 \\
\hline & $\mathrm{A}^{*} 02$ & $60.98(100 / 164)$ & $36.86(129 / 350)$ & $2.73(1.85,4.03) / 2$ & $<0.001$ \\
\hline & $\mathrm{A}^{*} 03$ & $18.90(31 / 164)$ & $22.29(78 / 350)$ & $0.81(0.51,1.29) / 2$ & 0.373 \\
\hline & $\mathrm{A}^{*} 09$ & $20.83(10 / 48)$ & $18.69(20 / 107)$ & $1.14(0.49,2.68) / 1$ & 0.775 \\
\hline & $\mathrm{A}^{*} 10$ & $8.33(4 / 48)$ & $27.10(29 / 107)$ & $0.24(0.08,0.74) / 1$ & 0.013 \\
\hline & $\mathrm{A}^{*} 11$ & $6.71(11 / 164)$ & $10.29(36 / 350)$ & $0.82 \mathrm{r}(0.20,3.32) / 2$ & 0.776 \\
\hline & $A^{*} 23$ & $7.76(9 / 116)$ & $9.88(24 / 243)$ & $0.77(0.34,1.71) / 1$ & 0.517 \\
\hline & $\mathrm{A}^{*} 24$ & $18.10(21 / 116)$ & $17.28(42 / 243)$ & $1.06(0.59,1.89) / 1$ & 0.849 \\
\hline & $\mathrm{A}^{*} 25$ & $0.00(0 / 116)$ & $3.70(9 / 243)$ & $0.11(0.01,1.84) / 1$ & 0.123 \\
\hline & $A^{*} 26$ & $7.76(9 / 116)$ & $8.23(20 / 243)$ & $0.94(0.41,2.13) / 1$ & 0.878 \\
\hline & $A^{*} 28$ & $6.25(3 / 48)$ & $11.21(12 / 107)$ & $0.53(0.14,1.96) / 1$ & 0.340 \\
\hline & $\mathrm{A}^{*} 29$ & $6.10(10 / 164)$ & $9.43(33 / 350)$ & $0.64(0.31,1.31) / 2$ & 0.222 \\
\hline
\end{tabular}


TABLE 3: Continued.

\begin{tabular}{|c|c|c|c|c|c|}
\hline Ethnicity & Allele & Patients\% $(n / N)$ & Controls $\%(n / N)$ & OR (95\% CI)/article number & $P$ \\
\hline & $\mathrm{A}^{*} 30$ & $12.93(15 / 116)$ & $15.23(37 / 243)$ & $0.83(0.43,1.58) / 1$ & 0.564 \\
\hline & $A^{*} 31$ & $5.17(6 / 116)$ & $9.47(23 / 243)$ & $0.52(0.21,1.32) / 1$ & 0.169 \\
\hline & $A^{*} 32$ & $3.45(4 / 116)$ & $8.23(20 / 243)$ & $0.40(0.13,1.19) / 1$ & 0.100 \\
\hline & $A^{*} 33$ & $5.17(6 / 116)$ & $6.17(15 / 243)$ & $0.83(0.31,2.20) / 1$ & 0.706 \\
\hline & $\mathrm{A}^{*} 34$ & $0.00(0 / 116)$ & $3.29(8 / 243)$ & $0.12(0.01,2.08) / 1$ & 0.145 \\
\hline & $A^{*} 36$ & $0.00(0 / 116)$ & $1.65(4 / 243)$ & $0.23(0.01,4.28) / 1$ & 0.323 \\
\hline & $A^{*} 66$ & $2.59(3 / 116)$ & $0.82(2 / 243)$ & $3.20(0.53,19.41) / 1$ & 0.206 \\
\hline & $A^{*} 68$ & $15.52(18 / 116)$ & $9.05(22 / 243)$ & $1.85(0.95,3.59) / 1$ & 0.072 \\
\hline & $A^{*} 69$ & $0.00(0 / 116)$ & $0.82(2 / 243)$ & $0.41(0.02,8.71) / 1$ & 0.571 \\
\hline & $\mathrm{A}^{*} 80$ & $0.00(0 / 116)$ & $0.41(1 / 243)$ & $0.69(0.03,17.16) / 1$ & 0.823 \\
\hline & $\mathrm{Aw}^{*} 19$ & $0.00(0 / 48)$ & $0.93(1 / 107)$ & $0.73(0.03,18.29) / 1$ & 0.849 \\
\hline & $\mathrm{Aw}^{*} 23$ & $2.08(1 / 48)$ & $4.67(5 / 107)$ & $0.43(0.05,3.82) / 1$ & 0.452 \\
\hline & $\mathrm{Aw}^{*} 24$ & $0.00(0 / 48)$ & $2.80(3 / 107)$ & $0.31(0.02,6.08) / 1$ & 0.439 \\
\hline & $\mathrm{Aw}^{*} 30$ & $20.83(10 / 48)$ & $28.04(30 / 107)$ & $0.68(0.30,1.52) / 1$ & 0.345 \\
\hline & $\mathrm{Aw}^{*} 31$ & $16.67(8 / 48)$ & $4.67(5 / 107)$ & $4.08(1.26,13.22) / 1$ & 0.019 \\
\hline & $\mathrm{Aw}^{*} 32$ & $4.17(2 / 48)$ & $8.41(9 / 107)$ & $0.47(0.10,2.28) / 1$ & 0.351 \\
\hline & $\mathrm{Aw}^{*} 33$ & $6.25(3 / 48)$ & $4.67(5 / 107)$ & $1.36(0.31,5.94) / 1$ & 0.683 \\
\hline
\end{tabular}

$N$ : total number of subjects, $n$ : positive number of subjects, OR: odds ratio, CI: confidence interval, article number: total number of the articles relevant to the association between vitiligo and HLA-A alleles, r: random effects model, and the others: fixed effects model.

author, publication year, study design, country, ethnicity, characteristics of study population, numbers of cases and controls, typing methods, frequencies of HLA-A alleles, and study quality.

The methodological quality of included studies was assessed using the criteria proposed by Chalmers et al. [30], which consists of three major aspects: selection of subjects, comparability between groups, and outcome presented. The selected studies were rated on an ordinal star scoring scale from one to nine, with scores of five or more stars representing high quality [31].

2.4. Statistical Analysis. The chi-square and Fisher's exact tests were applied to compare the frequencies of HLA-A alleles in patients with vitiligo and controls to confirm the associated alleles, with significance set at $P<0.05$. Metaanalysis of the association between HLA-A alleles and vitiligo was performed using two different approaches: a fixed effects model and a random effects model. Heterogeneity among studies was evaluated through the chi-square test and $I^{2}$ statistic, and $P<0.10$ or $I^{2}>50 \%$ was considered statistically significant. The pooled ORs and 95\% confidence intervals (CIs) were calculated using either the random effects model when heterogeneity was confirmed or the fixed effects model when heterogeneity was absent. The test for overall effect was conducted using $Z$-scores, with significance set at $P<0.05$. Subgroup analyses were conducted according to ethnicity, clinical type, and typing methods. Sensitivity was analyzed by omitting each study at each step to assess whether any single study had a significant influence on the pooled OR. Finally, publication bias was assessed by Begg's funnel plots and Egger's linear regression test, and the significance level was set at $P<0.05$. All statistical analyses were performed using SPSS software (version 19.0; SPSS Institute, Chicago, USA) and STATA software (version 12.0; Stata Corporation, College Station, TX, USA). All tests were two-sided.

\section{Results}

3.1. Literature Search. Initially, a total of 1158 records were identified through database searches. After removing duplicates and screening titles and abstracts, 98 full-text articles were reviewed and 18 studies [10-23, 25, 26, 32, 33] finally met the inclusion criteria. One additional study [24] was identified from a review of the reference lists. Altogether, 19 case-control studies were included in this meta-analysis. The procedure of literature search and study selection is shown in Figure 1.

3.2. Study Characteristics. The main characteristics of the included studies are summarized in Table 1 . These 19 studies comprised 3042 patients with vitiligo and 5614 controls. Twelve studies $[10-17,19,22,24,32]$ were conducted in Asians, four [18, 20, 21, 23] were performed in Europeans, two $[26,33]$ were investigated in Americans, and the remaining one [25] was carried out in mixed populations. HLA-A typing methods such as lymphocytotoxicity test (LCT) [11, 13, 1526], polymerase chain reaction sequence-specific oligonucleotides (PCR-SSO) [33], PCR sequence-specific oligonucleotide probes (PCR-SSOP) [32], and PCR sequence-specific primers (PCR-SSP) $[10,12,14]$ were reported in the studies. In total, 33 HLA-A alleles were involved. The results of the chisquare and Fisher's exact tests indicated that 18 alleles were 


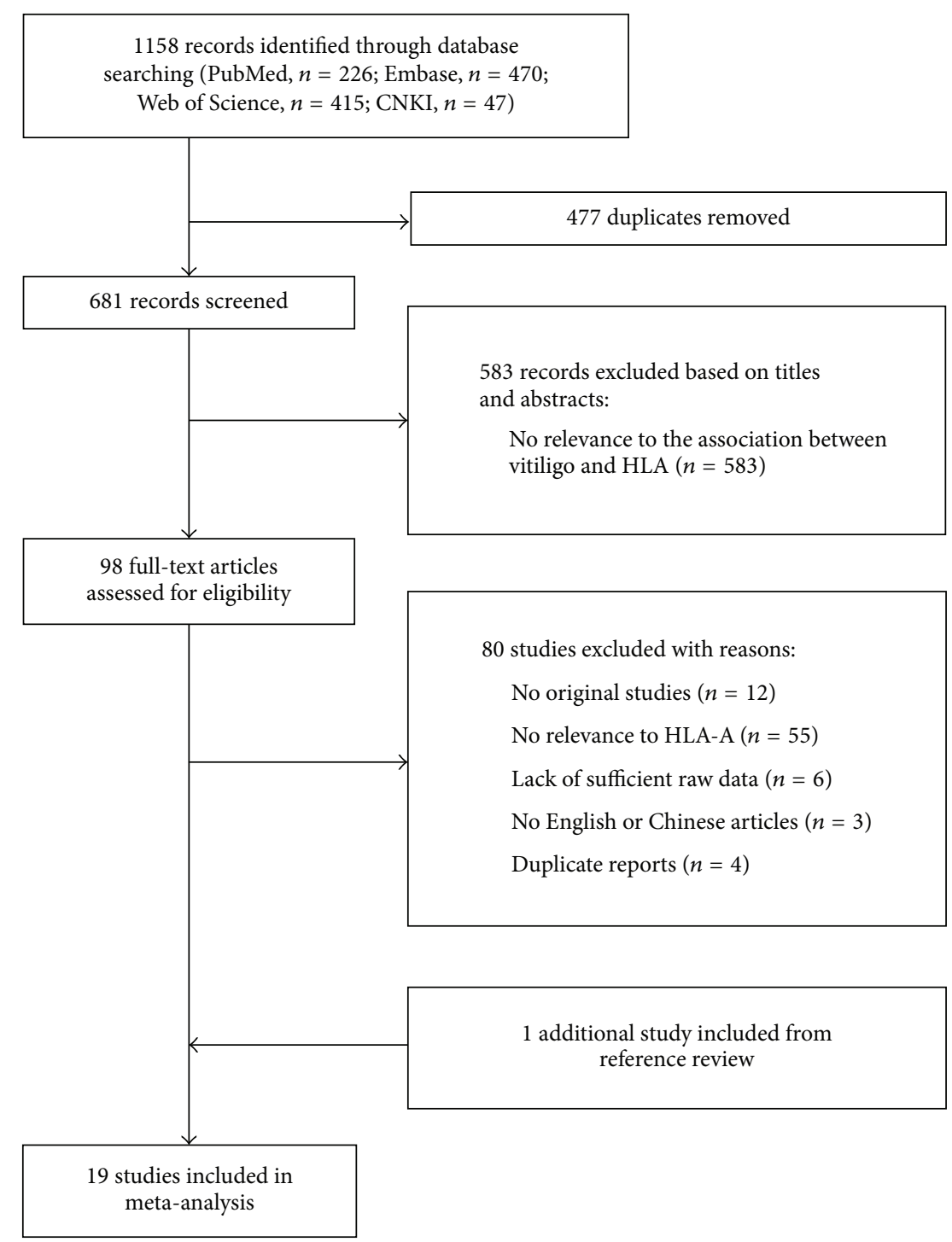

FIGURE 1: Flow diagram of study selection. HLA: human leukocyte antigen.

associated with vitiligo and 31 were unassociated. Sixteen alleles were disputed. According to the quality assessment criteria [30, 31], all the 19 studies [10-26, 32, 33] were of high quality with scores between five and nine stars.

3.3. Association between Vitiligo and Human Leukocyte Antigen-A. The general information on the association of vitiligo with HLA-A is given in Table 2. Among the 33 HLA-A alleles included in the pooled analysis, three (HLA-A* $02, A^{*} 33$, and $\left.\mathrm{Aw}^{*} 31\right)$ were significantly associated with increased risk of vitiligo, while two (HLA-A* 09 and $\left.\mathrm{Aw}^{*}{ }^{*} 19\right)$ were associated with decreased risk. HLA-A* 02 was reported in 15 studies. The pooled OR calculated with the random effects model was 1.52 (95\% CI: 1.21-1.90, $P<0.001$ ) (Figure 2), and the heterogeneity was significant $\left(I^{2}=61.7 \%, P=0.001\right)$.

The rest 28 alleles were not associated with vitiligo, of which HLA-A* 01 and $\mathrm{A}^{*} 03$ were each involved in more than 10 studies. The pooled ORs calculated with the random effects model were 0.95 (95\% CI: $0.66-1.35, P=0.761)$ and 1.45 (95\% CI: $0.99-2.13, P=0.056$ ), respectively (Figures 3 and 4). Significant heterogeneity among the studies was found $\left(I^{2}=55.8 \%, P=0.012\right.$, and $I^{2}=51.3 \%, P=0.024$, resp. $)$.

3.4. Subgroup Analysis according to Ethnicity. Table 3 presents the results of subgroup analysis based on ethnicity. Of the 26 HLA-A alleles studied in Asian patients with vitiligo, four (HLA-A* $03, A^{*} 10, A^{*} 25$, and $A^{*} 33$ ) were significantly associated with increased risk of vitiligo and three (HLA$\mathrm{A}^{*} 09, \mathrm{~A}^{*} 66$, and $\left.\mathrm{Aw}^{*} 19\right)$ were associated with decreased risk. The remaining 19 alleles were not associated with vitiligo. Among the 12 HLA-A alleles reported in European cases, one (HLA-A*02) was significantly associated with increased risk of vitiligo, and one (HLA-A*01) was associated with decreased risk. The remaining 10 alleles were not associated. For American cases, 29 HLA-A alleles were studied. Two alleles (HLA-A* 02 and $\mathrm{Aw}^{*}$ 31) were significantly associated 


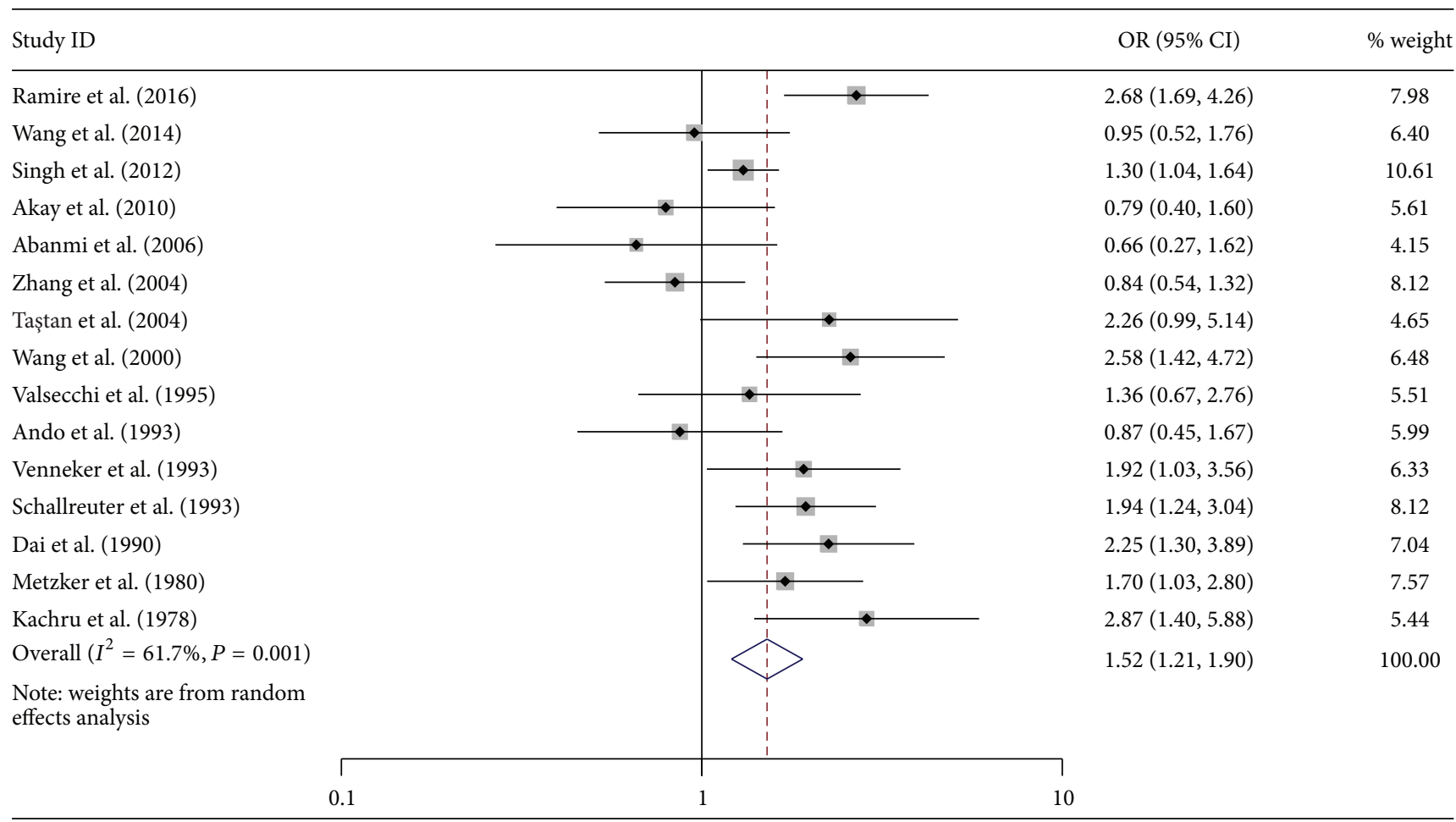

FIGURE 2: Forest plot of 15 included studies on the association between vitiligo and human leukocyte antigen- (HLA-) $A^{*} 02$. OR: odds ratio; CI: confidence interval.

\begin{tabular}{|c|c|c|c|c|}
\hline \multicolumn{3}{|l|}{ Study ID } & \multirow{2}{*}{$\begin{array}{c}\text { OR }(95 \% \mathrm{CI}) \\
1.15(0.65,2.04)\end{array}$} & \multirow{2}{*}{$\begin{array}{c}\text { \% weight } \\
12.53\end{array}$} \\
\hline Ramire et al. (2016) & & $\bullet$ & & \\
\hline Akay et al. (2010) & & & $0.96(0.36,2.54)$ & 7.76 \\
\hline Abanmi et al. (2006) & & $\longrightarrow$ & $3.35(0.63,17.74)$ & 3.68 \\
\hline Taştan et al. (2004) & & $\rightarrow$ & $1.23(0.46,3.26)$ & 7.74 \\
\hline Zhang et al. (2004) & $\rightarrow$ & - & $0.83(0.54,1.29)$ & 14.54 \\
\hline Wang et al. (2000) & 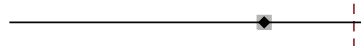 & - & $0.52(0.09,2.89)$ & 3.49 \\
\hline Valsecchi et al. (1995) & $\longrightarrow$ & - & $0.42(0.15,1.23)$ & 6.97 \\
\hline Schallreuter et al. (1993) & $\bullet$ & & $0.47(0.27,0.81)$ & 12.84 \\
\hline Dai et al. (1990) & $\longrightarrow$ & & $0.72(0.27,1.93)$ & 7.65 \\
\hline Metzker et al. (1980) & $\rightarrow$ & & $0.88(0.52,1.50)$ & 13.11 \\
\hline Kachru et al. (1978) & & $\longrightarrow$ & $3.41(1.55,7.52)$ & 9.71 \\
\hline Overall $\left(I^{2}=55.8 \%, P=0.012\right)$ & & & $0.95(0.66,1.35)$ & 100.00 \\
\hline $\begin{array}{l}\text { Note: weights are from random } \\
\text { effects analysis }\end{array}$ & & & & \\
\hline & $\Gamma$ & $\neg$ & & \\
\hline & 0.1 & 10 & & \\
\hline
\end{tabular}

FIGURE 3: Forest plot of 11 included studies on the association between vitiligo and human leukocyte antigen- (HLA-) A*01. OR: odds ratio; $\mathrm{CI}$ : confidence interval.

with increased risk of vitiligo, and one (HLA-A*10) was associated with decreased risk. The other 26 alleles were not associated.

Twelve alleles (HLA-A*01, A*02, A*03, A*09, A*10, A*11, $A^{*} 28, A^{*} 29, A^{*} 30, A^{*} 31, A^{*} 32$, and $\left.A^{*} 33\right)$ were common to Asians, Europeans, and Americans, but six (HLA-A* ${ }^{*}$,
$A^{*} 02, A^{*} 03, A^{*} 09, A^{*} 10$, and $\left.A^{*} 33\right)$ of them were inconsistent in their association with vitiligo.

3.5. Subgroup Analysis according to Clinical Type. Table 4 demonstrates the results of subgroup analysis based on clinical type. Among the 26 HLA-A alleles studied in patients 


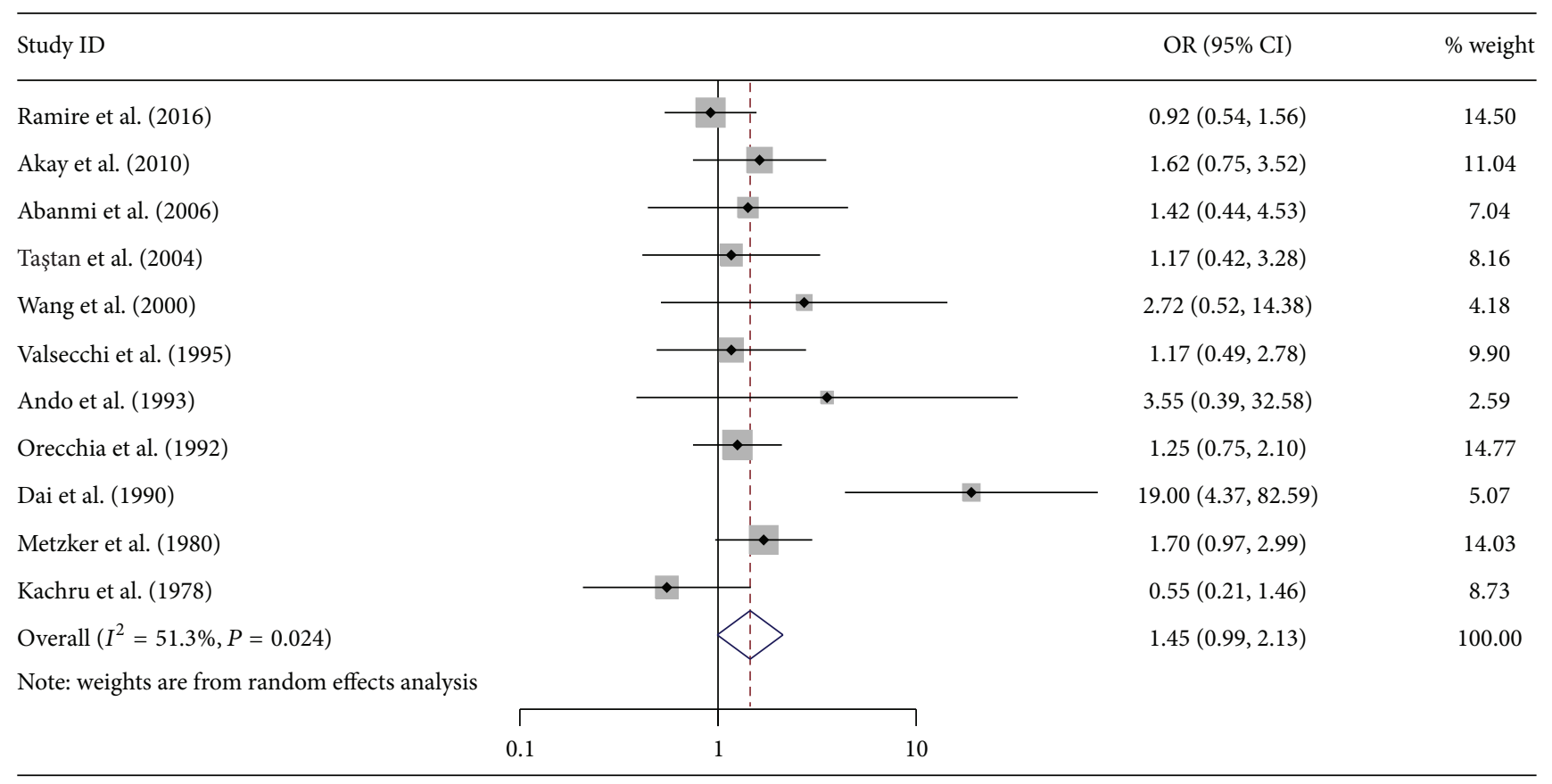

FIGURE 4: Forest plot of 11 included studies on the association between vitiligo and human leukocyte antigen- (HLA-) $\mathrm{A}^{*} 03$. OR: odds ratio; CI: confidence interval.

with nonsegmental vitiligo, three (HLA-A ${ }^{*} 02, \mathrm{~A}^{*} 03$, and $\left.A^{*} 33\right)$ were significantly associated with increased risk of nonsegmental vitiligo and one (HLA-A*19) was associated with decreased risk. The remaining 22 alleles were not associated with nonsegmental vitiligo. Of the seven HLA-A alleles reported in cases of segmental vitiligo, two (HLA-A ${ }^{*} 02$ and $A^{*} 03$ ) were significantly associated with increased risk of segmental vitiligo and the remaining five were not associated.

Seven alleles (HLA-A ${ }^{*} 01, A^{*} 02, A^{*} 03, A^{*} 09, A^{*} 10, A^{*} 11$, and $\left.A^{*} 28\right)$ were common to both types of vitiligo. Moreover, all of them were consistent in their association with vitiligo.

3.6. Subgroup Analysis according to Typing Methods. Table 5 indicates the results of subgroup analysis based on typing methods. Two kinds of HLA-A typing methods were involved: serological methods (LCT) and molecular methods (PCR-SSO, PCR-SSOP, and PCR-SSP). Of the 31 HLA-A alleles detected by serological methods, four (HLA-A ${ }^{*} 02$, $\mathrm{A}^{*} 03, \mathrm{~A}^{*} 31$, and $\left.\mathrm{Aw}^{*} 31\right)$ were significantly associated with increased risk of vitiligo and one (HLA-A* 09 ) was associated with decreased risk. The remaining 26 alleles were not associated with vitiligo. Among the $21 \mathrm{HLA}-\mathrm{A}$ alleles detected by molecular methods, two (HLA-A ${ }^{*} 36$ and $\left.A^{*}{ }^{*} 19\right)$ were significantly associated with increased risk of vitiligo and the remaining 19 were not associated.

Nineteen alleles (HLA-A ${ }^{*} 01, A^{*} 02, A^{*} 03, A^{*} 11, A^{*} 23$, $A^{*} 24, A^{*} 25, A^{*} 26, A^{*} 29, A^{*} 30, A^{*} 31, A^{*} 32, A^{*} 33, A^{*} 36$, $\mathrm{A}^{*} 43, \mathrm{~A}^{*} 66, \mathrm{~A}^{*} 68, \mathrm{~A}^{*} 80$, and $\left.\mathrm{Aw}^{*} 19\right)$ were common to both typing methods. However, five $\left(\mathrm{HLA}^{*} \mathrm{~A}^{*} 02, \mathrm{~A}^{*} 03\right.$, $A^{*} 31, A^{*} 36$, and $\left.A w^{*} 19\right)$ of them were inconsistent in their association with vitiligo.
3.7. Sensitivity Analysis and Publication Bias. Among the 33 alleles investigated in 19 included studies, HLA-A*01, $A^{*} 02$, and $\mathrm{A}^{*} 03$ were reported in more than 10 studies and were therefore chosen for sensitivity analysis and assessment of publication bias. As shown in Figure 5, sensitivity analyses indicated that no single study substantially influenced the pooled ORs qualitatively (data not shown). Begg's funnel plots and Egger's test were performed to assess publication bias. No obvious publication bias was found in the results (HLA-A ${ }^{*} 01, \mathrm{~A}^{*} 02$, and $\mathrm{A}^{*} 03: P=0.566,0.749$, and 0.160 , resp.) (Figure 6).

\section{Discussion}

In the present study, we performed a meta-analysis to comprehensively evaluate the association between vitiligo and 33 HLA-A alleles. Nineteen case-control studies [10-26, 32, 33] with a total of 3042 vitiligo cases and 5614 controls were finally identified from four databases and reference review. Overall, three alleles (HLA-A*02, $A^{*} 33$, and $\mathrm{Aw}^{*} 31$ ) were significantly associated with increased risk of vitiligo, while two (HLA-A* 09 and $\mathrm{Aw}^{*}{ }^{*}$ ) ) were associated with decreased risk. The association between HLA-A* 02 and vitiligo was consistent with the results of Liu et al. [29]. In addition, the remaining 28 alleles were not associated with vitiligo.

There were 12 alleles common to three ethnicities (Asians, Europeans, and Americans), seven alleles common to both types of vitiligo (nonsegmental and segmental), and 19 alleles common to both typing methods (serological and molecular). In the subgroup analysis by ethnicity, the association of six alleles was consistent in three populations, while that 
TABLE 4: Association between vitiligo and human leukocyte antigen- (HLA-) A alleles in terms of clinical type.

\begin{tabular}{|c|c|c|c|c|c|}
\hline Clinical type & Allele & Patients\% $(n / N)$ & Controls\% $(n / N)$ & OR $(95 \% \mathrm{CI}) /$ article number & $P$ \\
\hline \multirow{26}{*}{ Nonsegmental } & $\mathrm{A}^{*} 01$ & $29.84(94 / 315)$ & $32.45(355 / 1094)$ & $0.81(0.57,1.14) / 5$ & 0.226 \\
\hline & $A^{*} 02$ & $23.36(425 / 1819)$ & $33.08(1241 / 3751)$ & $1.46 \mathrm{r}(1.01,2.10) / 9$ & 0.042 \\
\hline & $\mathrm{A}^{*} 03$ & $20.09(85 / 423)$ & $12.86(241 / 1874)$ & $1.98 \mathrm{r}(1.02,3.81) / 7$ & 0.042 \\
\hline & $\mathrm{A}^{*} 09$ & $21.19(50 / 236)$ & $25.84(255 / 987)$ & $0.70(0.49,1.00 / 4$ & 0.052 \\
\hline & $A^{*} 10$ & $13.62(29 / 213)$ & $10.01(70 / 699)$ & $2.09 \mathrm{r}(0.83,5.28) / 4$ & 0.117 \\
\hline & $\mathrm{A}^{*} 11$ & $16.15(42 / 260)$ & $15.73(218 / 1386)$ & $0.90(0.61,1.34) / 5$ & 0.607 \\
\hline & $A^{*} 19$ & $22.50(18 / 80)$ & $43.75(35 / 80)$ & $0.28(0.13,0.63) / 2$ & 0.002 \\
\hline & $A^{*} 23$ & $5.13(4 / 78)$ & $9.88(24 / 243)$ & $0.49(0.17,1.47) / 1$ & 0.204 \\
\hline & $A^{*} 24$ & $25.64(30 / 117)$ & $46.25(364 / 787)$ & $0.72(0.44,1.17) / 2$ & 0.180 \\
\hline & $A^{*} 25$ & $19.19(33 / 172)$ & $6.46(32 / 495)$ & $1.18 \mathrm{r}(0.03,47.18) / 2$ & 0.930 \\
\hline & $A^{*} 26$ & $7.64(119 / 1558)$ & $13.32(313 / 2349)$ & $0.91 \mathrm{r}(0.58,1.43) / 4$ & 0.676 \\
\hline & $A^{*} 28$ & $13.29(19 / 143)$ & $7.35(44 / 599)$ & $1.00(0.50,1.98) / 3$ & 0.996 \\
\hline & $A^{*} 29$ & $7.21(8 / 111)$ & $8.60(59 / 686)$ & $0.75(0.34,1.62) / 2$ & 0.460 \\
\hline & $A^{*} 30$ & $13.87(53 / 382)$ & $7.24(110 / 1520)$ & $1.84 \mathrm{r}(0.73,4.62) / 6$ & 0.196 \\
\hline & $\mathrm{A}^{*} 31$ & $4.02(64 / 1591)$ & $6.66(186 / 2792)$ & $1.23 \mathrm{r}(0.54,2.79) / 5$ & 0.626 \\
\hline & $A^{*} 32$ & $3.60(4 / 111)$ & $8.02(55 / 686)$ & $0.41 \mathrm{r}(0.01,15.55) / 2$ & 0.628 \\
\hline & $A^{*} 33$ & $23.05(336 / 1458)$ & $9.02(180 / 1996)$ & $2.34(1.91,2.86) / 3$ & $<0.001$ \\
\hline & $\mathrm{A}^{*} 34$ & $0.00(0 / 78)$ & $3.29(8 / 243)$ & $0.18(0.01,3.09) / 1$ & 0.235 \\
\hline & $A^{*} 36$ & $0.94(2 / 212)$ & $2.24(12 / 535)$ & $0.54(0.15,1.94) / 3$ & 0.344 \\
\hline & $A^{*} 38$ & $0.00(0 / 40)$ & $2.50(1 / 40)$ & $0.33(0.01,8.22) / 1$ & 0.495 \\
\hline & $A^{*} 43$ & $25.37(34 / 134)$ & $36.64(107 / 292)$ & $0.75(0.47,1.22) / 2$ & 0.251 \\
\hline & $A^{*} 66$ & $3.49(6 / 172)$ & $8.89(44 / 495)$ & $0.83 \mathrm{r}(0.03,23.79) / 2$ & 0.914 \\
\hline & $A^{*} 68$ & $16.07(229 / 1425)$ & $13.33(207 / 1553)$ & $1.21(0.98,1.48) / 2$ & 0.072 \\
\hline & $A^{*} 69$ & $0.00(0 / 78)$ & $0.82(2 / 243)$ & $0.62(0.03,12.95) / 1$ & 0.755 \\
\hline & $\mathrm{A}^{*} 80$ & $1.69(2 / 118)$ & $1.77(5 / 283)$ & $0.56(0.12,2.66) / 2$ & 0.468 \\
\hline & $\mathrm{Aw}^{*} 33$ & $12.82(5 / 39)$ & $13.79(75 / 544)$ & $0.92(0.35,2.43) / 1$ & 0.866 \\
\hline \multirow{7}{*}{ Segmental } & $\mathrm{A}^{*} 01$ & $6.67(2 / 30)$ & $9.48(11 / 116)$ & $0.68(0.14,3.26) / 1$ & 0.631 \\
\hline & $\mathrm{A}^{*} 02$ & $67.27(37 / 55)$ & $47.22(102 / 216)$ & $2.34(1.25,4.38) / 2$ & 0.008 \\
\hline & $\mathrm{A}^{*} 03$ & $7.27(4 / 55)$ & $1.85(4 / 216)$ & $4.09(1.08,15.51) / 2$ & 0.038 \\
\hline & $\mathrm{A}^{*} 09$ & $26.67(8 / 30)$ & $32.76(38 / 116)$ & $0.75(0.30,1.83) / 1$ & 0.523 \\
\hline & $\mathrm{A}^{*} 10$ & $7.27(4 / 55)$ & $3.70(8 / 216)$ & $2.03(0.59,7.01) / 2$ & 0.261 \\
\hline & $\mathrm{A}^{*} 11$ & $33.33(10 / 30)$ & $30.17(35 / 116)$ & $1.16(0.49,2.72) / 1$ & 0.738 \\
\hline & $A^{*} 28$ & $6.67(2 / 30)$ & $5.17(6 / 116)$ & $1.31(0.25,6.84) / 1$ & 0.749 \\
\hline
\end{tabular}

$N$ : total number of subjects, $n$ : positive number of subjects, OR: odds ratio, CI: confidence interval, article number: total number of the articles relevant to the association between vitiligo and HLA-A alleles, r: random effects model, and the others: fixed effects model.

of the remaining six alleles (HLA- $A^{*} 01, A^{*} 02, A^{*} 03, A^{*} 09$, $\mathrm{A}^{*} 10$, and $\mathrm{A}^{*} 33$ ) was inconsistent. The possible reasons for these inconsistencies might be the difference in ethnicity or the comparatively small number of included studies for some alleles. Subgroup analysis by clinical type showed that the association of all seven alleles (HLA-A*01, $A^{*} 02, A^{*} 03$, $\mathrm{A}^{*} 09, \mathrm{~A}^{*} 10, \mathrm{~A}^{*} 11$, and $\mathrm{A}^{*} 28$ ) was consistent in both types of vitiligo. It suggests that the association between vitiligo and these alleles may be independent of clinical type. However, we should interpret this association with great caution because only two studies $[16,24]$ presented relevant data on segmental vitiligo and were included in this meta-analysis. HLA-A typing is critical for the accuracy of test results. In the 19 studies of this meta-analysis, serological and molecular methods were involved, and the latter had higher resolution than the former. In the subgroup analysis by typing methods, the association of five alleles (HLA-A* $02, \mathrm{~A}^{*} 03, \mathrm{~A}^{*} 31, \mathrm{~A}^{*} 36$, and $\left.A w^{*} 19\right)$ was inconsistent in both typing methods. It suggests that the association of vitiligo with these five alleles may vary in terms of typing methods.

Among the 33 HLA-A alleles in the current meta-analysis, only three alleles (HLA-A ${ }^{*} 01, \mathrm{~A}^{*} 02$, and $\mathrm{A}^{*} 03$ ) were reported in more than 10 studies. There was obvious heterogeneity among the studies for each allele, which might be caused by the differences in ethnicity, clinical type, and typing methods. However, sensitivity analyses indicated that the results for these three alleles were statistically reliable, and no publication bias was found based on the funnel plot analyses and Egger's tests. The association between the remaining 30 alleles and vitiligo needs to be further studied.

This study has some limitations. First, the meta-analysis only included published studies. Second, vitiligo may be 
TABLE 5: Association between vitiligo and human leukocyte antigen- (HLA-) A alleles in terms of typing methods.

\begin{tabular}{|c|c|c|c|c|c|}
\hline Typing methods & Allele & Patients\% $(n / N)$ & Controls $\%(n / N)$ & OR (95\% CI)/article number & $P$ \\
\hline \multirow{31}{*}{ Serological } & $\mathrm{A}^{*} 01$ & $15.69(91 / 580)$ & $23.77(444 / 1868)$ & $0.95 \mathrm{r}(0.58,1.56) / 9$ & 0.840 \\
\hline & $\mathrm{A}^{*} 02$ & $55.92(373 / 667)$ & $44.21(1377 / 3115)$ & $1.64 \mathrm{r}(1.26,2.13) / 11$ & $<0.001$ \\
\hline & $\mathrm{A}^{*} 03$ & $19.34(118 / 610)$ & $13.42(322 / 2400)$ & $1.58 \mathrm{r}(1.03,2.42) / 10$ & 0.035 \\
\hline & $\mathrm{A}^{*} 04$ & $3.85(2 / 52)$ & $3.00(3 / 100)$ & $1.29(0.21,7.99) / 1$ & 0.782 \\
\hline & $\mathrm{A}^{*} 09$ & $20.07(108 / 538)$ & $26.25(461 / 1756)$ & $0.70(0.54,0.90) / 8$ & 0.005 \\
\hline & $\mathrm{A}^{*} 10$ & $10.56(47 / 445)$ & $12.79(175 / 1368)$ & $1.11 \mathrm{r}(0.51,2.41) / 7$ & 0.786 \\
\hline & $\mathrm{A}^{*} 11$ & $17.99(93 / 517)$ & $14.61(294 / 2012)$ & $0.95(0.71,1.28) / 9$ & 0.751 \\
\hline & $\mathrm{A}^{*} 19$ & $22.50(18 / 80)$ & $43.75(35 / 80)$ & $0.19 \mathrm{r}(0.02,2.02) / 2$ & 0.170 \\
\hline & $A^{*} 23$ & $2.35(2 / 85)$ & $4.00(8 / 200)$ & $0.72(0.17,3.11) / 2$ & 0.662 \\
\hline & $\mathrm{A}^{*} 24$ & $25.00(31 / 124)$ & $48.12(358 / 744)$ & $0.79 \mathrm{r}(0.17,3.76) / 3$ & 0.767 \\
\hline & $A^{*} 25$ & $3.85(2 / 52)$ & $2.00(2 / 100)$ & $1.96(0.27,14.33) / 1$ & 0.507 \\
\hline & $A^{*} 26$ & $10.48(13 / 124)$ & $18.28(136 / 744)$ & $0.71(0.38,1.32) / 3$ & 0.282 \\
\hline & $A^{*} 28$ & $7.89(31 / 393)$ & $8.60(109 / 1268)$ & $0.74(0.46,1.17) / 6$ & 0.192 \\
\hline & $A^{*} 29$ & $4.94(12 / 243)$ & $7.34(89 / 1212)$ & $0.78(0.42,1.44) / 5$ & 0.434 \\
\hline & $A^{*} 30$ & $9.20(24 / 261)$ & $5.61(63 / 1123)$ & $1.55 \mathrm{r}(0.48,5.03) / 5$ & 0.468 \\
\hline & $\mathrm{A}^{*} 31$ & $14.26(15 / 105)$ & $8.28(90 / 1087)$ & $2.26(1.22,4.19) / 3$ & 0.009 \\
\hline & $A^{*} 32$ & $9.32(11 / 118)$ & $8.24(53 / 643)$ & $1.11(0.55,2.25) / 3$ & 0.774 \\
\hline & $A^{*} 33$ & $3.03(2 / 66)$ & $2.58(14 / 543)$ & $1.23(0.32,4.77) / 2$ & 0.763 \\
\hline & $A^{*} 36$ & $5.00(2 / 40)$ & $0.00(0 / 40)$ & $5.26(0.24,113.11) / 1$ & 0.289 \\
\hline & $\mathrm{A}^{*} 38$ & $0.00(0 / 40)$ & $2.50(1 / 40)$ & $0.33(0.01,8.22) / 1$ & 0.495 \\
\hline & $A^{*} 43$ & $5.00(2 / 40)$ & $0.00(0 / 40)$ & $5.26(0.24,113.11) / 1$ & 0.289 \\
\hline & $A^{*} 66$ & $0.00(0 / 52)$ & $2.00(2 / 100)$ & $0.38(0.02,7.96) / 1$ & 0.529 \\
\hline & $A^{*} 68$ & $5.88(5 / 85)$ & $2.50(5 / 200)$ & $2.36(0.67,8.40) / 2$ & 0.317 \\
\hline & $\mathrm{A}^{*} 80$ & $5.00(2 / 40)$ & $10.00(4 / 40)$ & $0.47(0.08,2.75) / 1$ & 0.405 \\
\hline & $\mathrm{Aw}^{*} 19$ & $0.00(0 / 48)$ & $0.93(1 / 107)$ & $0.73(0.03,18.29) / 1$ & 0.849 \\
\hline & $\mathrm{Aw}^{*} 23$ & $2.08(1 / 48)$ & $4.67(5 / 107)$ & $0.43(0.05,3.82) / 1$ & 0.452 \\
\hline & $\mathrm{Aw}^{*} 24$ & $0.00(0 / 48)$ & $2.80(3 / 107)$ & $0.31(0.02,6.08) / 1$ & 0.439 \\
\hline & $\mathrm{Aw}^{*} 30$ & $20.83(10 / 48)$ & $28.04(30 / 107)$ & $0.68(0.30,1.52) / 1$ & 0.345 \\
\hline & $\mathrm{Aw}^{*} 31$ & $16.67(8 / 48)$ & $4.67(5 / 107)$ & $4.08(1.26,13.22) / 1$ & 0.019 \\
\hline & $\mathrm{Aw}^{*} 32$ & $4.17(2 / 48)$ & $8.41(9 / 107)$ & $0.47(0.10,2.28) / 1$ & 0.351 \\
\hline & $\mathrm{Aw}^{*} 33$ & $9.20(8 / 87)$ & $12.29(80 / 651)$ & $1.03(0.46,2.30) / 2$ & 0.944 \\
\hline \multirow{21}{*}{ Molecular } & $\mathrm{A}^{*} 01$ & $52.15(158 / 303)$ & $47.07(233 / 495)$ & $0.94(0.66,1.33) / 2$ & 0.714 \\
\hline & $\mathrm{A}^{*} 02$ & $17.98(388 / 2158)$ & $17.74(333 / 1877)$ & $1.31 \mathrm{r}(0.84,2.05) / 4$ & 0.238 \\
\hline & $\mathrm{A}^{*} 03$ & $21.55(25 / 116)$ & $23.05(56 / 243)$ & $0.92(0.54,1.56) / 1$ & 0.752 \\
\hline & $\mathrm{A}^{*} 11$ & $6.03(7 / 116)$ & $12.76(31 / 243)$ & $0.44(0.19,1.03) / 1$ & 0.058 \\
\hline & $A^{*} 23$ & $7.76(9 / 116)$ & $9.88(24 / 243)$ & $0.77(0.34,1.71) / 1$ & 0.517 \\
\hline & $\mathrm{A}^{*} 24$ & $18.10(21 / 116)$ & $17.28(42 / 243)$ & $1.06(0.59,1.89) / 1$ & 0.849 \\
\hline & $A^{*} 25$ & $13.20(40 / 303)$ & $6.46(32 / 495)$ & $0.71 \mathrm{r}(0.03,19.23) / 2$ & 0.839 \\
\hline & $A^{*} 26$ & $7.91(163 / 2061)$ & $11.41(206 / 1805)$ & $0.83 \mathrm{r}(0.50,1.38) / 3$ & 0.479 \\
\hline & $A^{*} 29$ & $8.62(10 / 116)$ & $10.70(26 / 243)$ & $0.79(0.37,1.69) / 1$ & 0.540 \\
\hline & $A^{*} 30$ & $14.84(50 / 337)$ & $9.38(56 / 597)$ & $2.22 \mathrm{r}(0.72,6.83) / 3$ & 0.166 \\
\hline & $\mathrm{A}^{*} 31$ & $3.35(69 / 2061)$ & $5.48(99 / 1805)$ & $0.68 \mathrm{r}(0.30,1.55) / 3$ & 0.360 \\
\hline & $A^{*} 32$ & $3.45(4 / 116)$ & $8.23(20 / 243)$ & $0.40(0.13,1.19) / 1$ & 0.100 \\
\hline & $A^{*} 33$ & $22.84(428 / 1874)$ & $10.95(170 / 1553)$ & $1.56 \mathrm{r}(0.58,4.24) / 2$ & 0.379 \\
\hline & $\mathrm{A}^{*} 34$ & $0.00(0 / 116)$ & $3.29(8 / 243)$ & $0.12(0.01,2.08) / 1$ & 0.145 \\
\hline & $A^{*} 36$ & $0.00(0 / 303)$ & $2.42(12 / 495)$ & $0.12(0.02,0.91) / 2$ & 0.040 \\
\hline & $A^{*} 43$ & $37.43(70 / 187)$ & $42.46(107 / 252)$ & $0.81(0.55,1.19) / 1$ & 0.289 \\
\hline & $A^{*} 66$ & $3.30(10 / 303)$ & $8.89(44 / 495)$ & $0.70 \mathrm{r}(0.05,10.81) / 2$ & 0.798 \\
\hline & $A^{*} 68$ & $14.83(278 / 1874)$ & $13.33(207 / 1553)$ & $1.27 \mathrm{r}(0.76,2.13) / 2$ & 0.363 \\
\hline & $A^{*} 69$ & $0.00(0 / 116)$ & $0.82(2 / 243)$ & $0.41(0.02,8.71) / 1$ & 0.571 \\
\hline & $\mathrm{A}^{*} 80$ & $0.00(0 / 116)$ & $0.41(1 / 243)$ & $0.69(0.03,17.16) / 1$ & 0.823 \\
\hline & $\mathrm{Aw}^{*} 19$ & $0.74(13 / 1758)$ & $6.79(89 / 1310)$ & $0.10(0.06,0.18) / 1$ & $<0.001$ \\
\hline
\end{tabular}

$N$ : total number of subjects, $n$ : positive number of subjects, OR: odds ratio, CI: confidence interval, article number: total number of the articles relevant to the association between vitiligo and HLA-A alleles, r: random effects model, and the others: fixed effects model. 


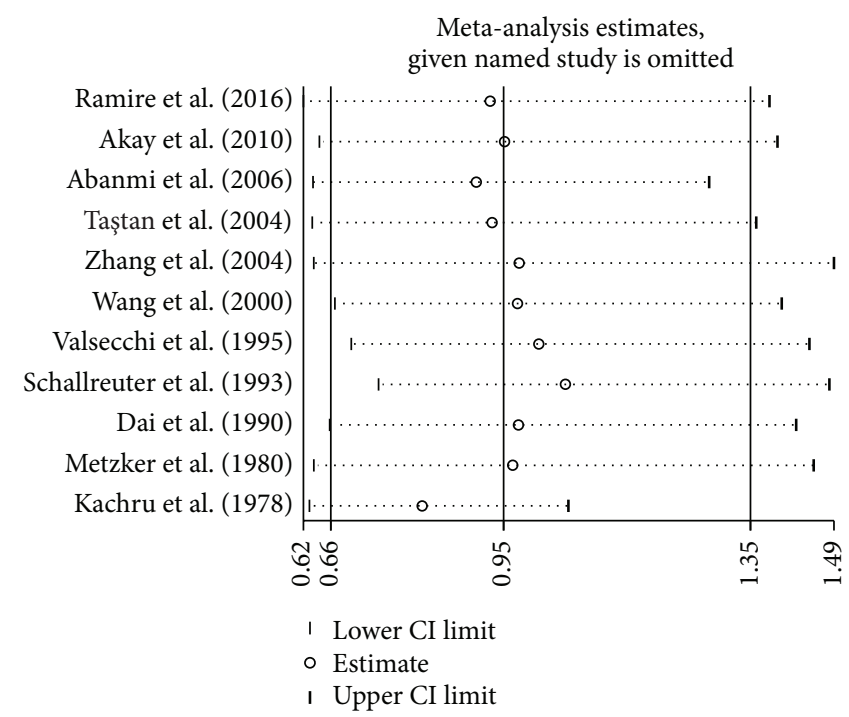

(a)

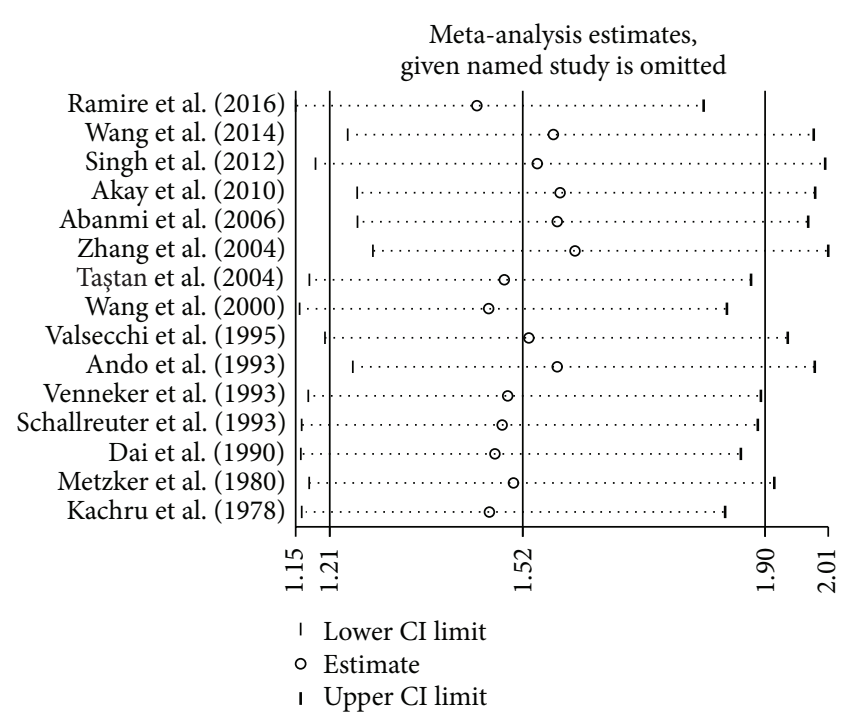

(b)

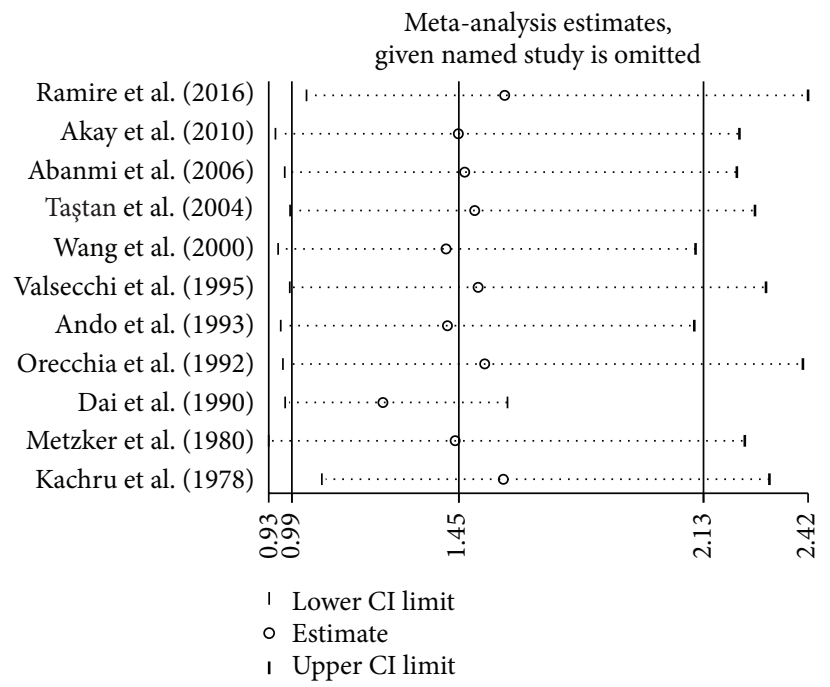

(c)

FiguRE 5: Influence of removing individual studies on adjusted effect estimates of (a) human leukocyte antigen- (HLA-) $A^{*} 01$, (b) A*02, and (c) $\mathrm{A}^{*}$ 03. Circles are effect estimates and horizontal dotted lines are $95 \%$ confidence intervals (CIs) for meta-analysis of the remaining studies. Vertical lines in the centers are the pooled effect estimates for all studies, respectively.

influenced by not only genetic factors but also environmental factors. The results of the meta-analysis should be interpreted cautiously owing to the lack of available data regarding vitiligo development and its relationship with genetic and environmental factors. Further studies may assess the possible gene-environment interactions in the association. Third, the relatively small samples of some HLA-A alleles limited the statistical power. Finally, we were not able to perform subgroup for each HLA-A allele due to the limited number of eligible studies, which might have affected the results. Therefore, more studies with larger sample sizes focusing on each HLA-A allele are needed to confirm these findings. Despite the limitations listed above, this study still has some strength. To the best of our knowledge, this is the first metaanalysis evaluating the association between vitiligo and a number of HLA-A alleles.

\section{Conclusion}

In summary, this meta-analysis suggests that HLA-A*02, $\mathrm{A}^{*} 33$, and $\mathrm{Aw}^{*} 31$ are associated with increased risk of vitiligo, while HLA-A*09 and Aw ${ }^{*} 19$ are associated with decreased risk of vitiligo. Moreover, the association of some alleles varies in terms of ethnicity and typing methods. However, further well-designed studies with larger sample sizes are still needed to confirm our findings. 


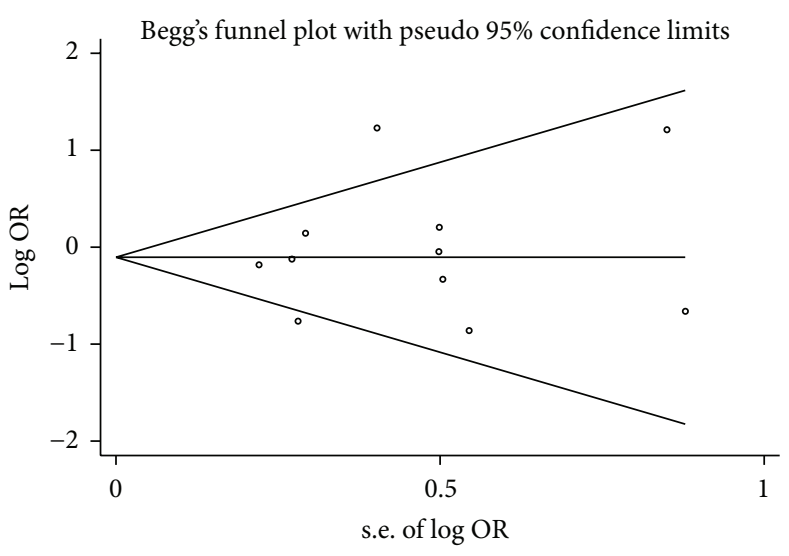

(a)

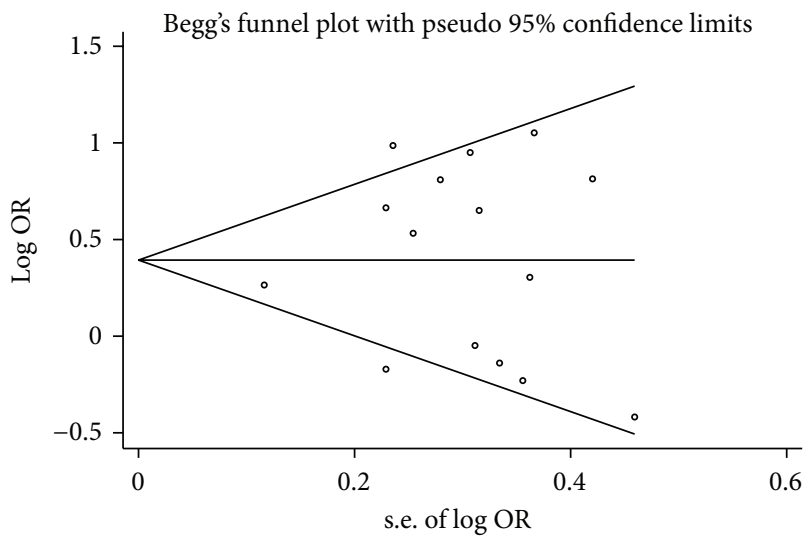

(b)

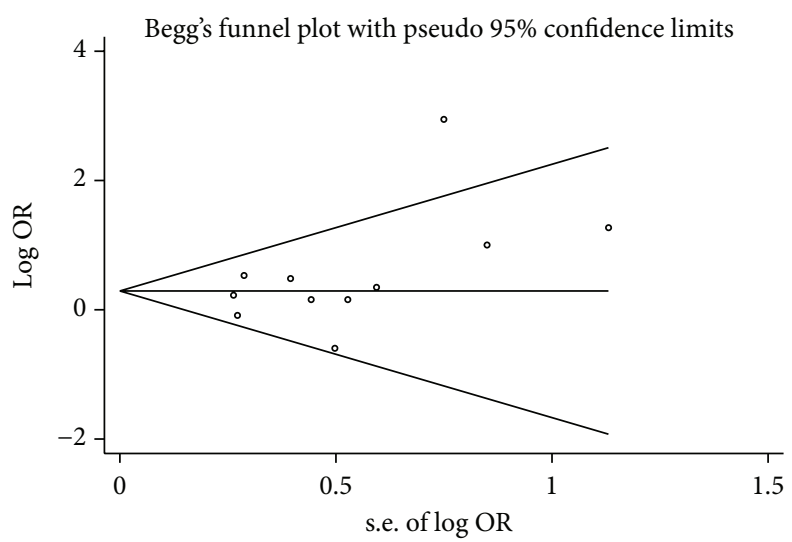

(c)

Figure 6: Funnel plots for meta-analysis of the association of vitiligo with (a) human leukocyte antigen- (HLA-) $A^{*} 01$, (b) $A^{*} 02$, and (c) $\mathrm{A}^{*}$ 03. $\log$ OR: natural logarithm of odds ratio.

\section{Competing Interests}

The authors declare that there is no conflict of interests regarding the publication of this paper.

\section{Acknowledgments}

The authors thank Dr. Jun Dong for providing some relevant data.

\section{References}

[1] C. Krüger and K. U. Schallreuter, "A review of the worldwide prevalence of vitiligo in children/adolescents and adults," International Journal of Dermatology, vol. 51, no. 10, pp. 1206-1212, 2012.

[2] T. Lotti and A. M. D'Erme, "Vitiligo as a systemic disease," Clinics in Dermatology, vol. 32, no. 3, pp. 430-434, 2014.

[3] N. Malhotra and M. Dytoc, "The pathogenesis of vitiligo," Journal of Cutaneous Medicine and Surgery, vol. 17, no. 3, pp. 153-172, 2013.

[4] R. A. Spritz, "The genetics of generalized vitiligo and associated autoimmune diseases," Journal of Dermatological Science, vol. 41, no. 1, pp. 3-10, 2006.
[5] A. Alkhateeb, P. R. Fain, A. Thody, D. C. Bennett, and R. A. Spritz, "Epidemiology of vitiligo and associated autoimmune diseases in Caucasian probands and their families," Pigment Cell Research, vol. 16, no. 3, pp. 208-214, 2003.

[6] E. H. Kemp, S. Emhemad, S. Akhtar, P. F. Watson, D. J. Gawkrodger, and A. P. Weetman, "Autoantibodies against tyrosine hydroxylase in patients with non-segmental (generalised) vitiligo," Experimental Dermatology, vol. 20, no. 1, pp. 35-40, 2011.

[7] R. Harning, J. Cui, and J.-C. Bystryn, "Relation between the incidence and level of pigment cell antibodies and disease activity in vitiligo," Journal of Investigative Dermatology, vol. 97, no. 6, pp. 1078-1080, 1991.

[8] P. P. Majumder, J. J. Nordlund, and S. K. Nath, "Pattern of familial aggregation of vitiligo," Archives of Dermatology, vol. 129, no. 8, pp. 994-998, 1993.

[9] S. K. Nath, P. P. Majumder, and J. J. Nordlund, "Genetic epidemiology of vitiligo: multilocus recessivity cross-validated," American Journal of Human Genetics, vol. 55, no. 5, pp. 981-990, 1994.

[10] H. N. Wang, Y. M. Qi, Y. F. Gao et al., "Frequencies of HLAA2 among patients with tumors, chronic hepatitis $\mathrm{C}$ and vitiligo in Henan Province," Journal of Zhengzhou University (Medical Sciences), vol. 49, no. 3, pp. 349-351, 2014 (Chinese). 
[11] B. N. Akay, M. Bozkir, Y. Anadolu, and S. Gullu, "Epidemiology of vitiligo, associated autoimmune diseases and audiological abnormalities: Ankara study of 80 patients in Turkey," Journal of the European Academy of Dermatology and Venereology, vol. 24, no. 10, pp. 1144-1150, 2010.

[12] J. Wang, Y.-M. Zhao, Y. Wang, Y. Xiao, Y.-K. Wang, and H.-D. Chen, "Association of HLA class I and II alleles with generalized vitiligo in Chinese Hans in north China," Chinese Journal of Medical Genetics, vol. 24, no. 2, pp. 221-223, 2007 (Chinese).

[13] A. Abanmi, F. A. Harthi, R. A. Baqami et al., "Association of HLA loci alleles and antigens in Saudi patients with vitiligo," Archives of Dermatological Research, vol. 298, no. 7, pp. 347-352, 2006.

[14] X.-J. Zhang, H.-S. Liu, Y.-H. Liang et al., "Association of HLA class I alleles with vitiligo in Chinese Hans," Journal of Dermatological Science, vol. 35, no. 2, pp. 165-168, 2004.

[15] H. B. Taştan, A. Akar, F. E. Orkunoglu, E. Arca, and A. Inal, "Association of HLA class I antigens and HLA class II alleles with Vitiligo in a Turkish population," Pigment Cell Research, vol. 17, no. 2, pp. 181-184, 2004.

[16] Y. Wang, Y. Xiao, Y. M. Zhao et al., "Study on association between vitiligo and HLA class I antigens," Chinese Journal of Dermatology, vol. 33, no. 6, pp. 33-35, 2000 (Chinese).

[17] M. N. Venkataram, A. G. White, W. A. Leeny, A. R. A. Suwaid, and A. S. Daar, "HLA antigens in Omani patients with vitiligo," Clinical and Experimental Dermatology, vol. 20, no. 1, pp. 35-37, 1995.

[18] R. Valsecchi, M. Bontempelli, T. Cainelli, P. Leghissa, and A. Di Landro, "Vitiligo is associated with a significant increase in HLA-DR6 and a decrease in DQw2 antigens in Northern Italian patients," Journal of the European Academy of Dermatology and Venereology, vol. 5, no. 1, pp. 9-14, 1995.

[19] A. Al-Fouzan, M. Al-Arbash, F. Fouad, S. A. Kaaba, M. A. Mousa, and S. A. Al-Harbi, "Study of HLA class I/II and T lymphocyte subsets in Kuwaiti vitiligo patients," European Journal of Immunogenetics, vol. 22, no. 2, pp. 209-213, 1995.

[20] G. T. Venneker, L. P. De Waal, W. Westerhof, J. D’Amaro, G. M. T. Schreuder, and S. S. Asghar, "HLA associations in vitiligo patients in the Dutch population," Disease Markers, vol. 11, no. 4, pp. 187-190, 1993.

[21] K. U. Schallreuter, C. Levenig, P. Kuhnl, C. Loliger, M. HohlTehari, and J. Berger, "Histocompatibility antigens in vitiligo: Hamburg study on 102 patients from Northern Germany," Dermatology, vol. 187, no. 3, pp. 186-192, 1993.

[22] I. Ando, H.-I. Chi, H. Nakagawa, and F. Otsuka, "Difference in clinical features and HLA antigens between familial and non-familial vitiligo of non-segmental type," British Journal of Dermatology, vol. 129, no. 4, pp. 408-410, 1993.

[23] G. Orecchia, L. Perfetti, P. Malagolt, F. Borghini, and Y. Kipervarg, "Vitiligo is associated with a significant increase in HLAA30, Cw6 AND DQw3 and a decrease in C4AQ0 in Northern Italian patients," Dermatology, vol. 185, no. 2, pp. 123-127, 1992.

[24] X. Dai, P. Y. Jin, L. Ma, and H. M. Cao, "A study on the association of HLA antigens with vitiligo," Chinese Journal of Dermatology, vol. 23, no. 1, pp. 31-33, 1990 (Chinese).

[25] A. Metzker, R. Zamir, E. Gazit, M. David, and E. J. Feuerman, "Vitiligo and the hla system," Dermatology, vol. 160, no. 2, pp. 100-105, 1980.

[26] R. B. Kachru, M. Telischi, and K. K. Mittal, "HLA antigens and vitiligo in an American black population," Tissue Antigens, vol. 12, no. 5, pp. 396-397, 1978.
[27] Y. Jin, T. Ferrara, K. Gowan et al., "Next-generation DNA re-sequencing identifies common variants of TYR and HLAA that modulate the risk of generalized vitiligo via antigen presentation," Journal of Investigative Dermatology, vol. 132, no. 6, pp. 1730-1733, 2012.

[28] Y. Jin, M. Hayashi, P. R. Fain et al., "Major association of vitiligo with HLA-A* 02: 01 in Japanese," Pigment Cell and Melanoma Research, vol. 28, no. 3, pp. 360-362, 2015.

[29] J.-B. Liu, M. Li, H. Chen et al., "Association of vitiligo with HLA-A2: a meta-analysis," Journal of the European Academy of Dermatology and Venereology, vol. 21, no. 2, pp. 205-213, 2007.

[30] T. C. Chalmers, H. Smith Jr., B. Blackburn et al., "A method for assessing the quality of a randomized control trial," Controlled Clinical Trials, vol. 2, no. 1, pp. 31-49, 1981.

[31] L. Yan, J.-M. Wang, and K. Zeng, "Association between HLA-DRB1 polymorphisms and pemphigus vulgaris: a metaanalysis," British Journal of Dermatology, vol. 167, no. 4, pp. 768777, 2012.

[32] A. Singh, P. Sharma, H. K. Kar et al., "HLA alleles and amino-acid signatures of the peptide-binding pockets of HLA molecules in vitiligo," Journal of Investigative Dermatology, vol. 132, no. 1, pp. 124-134, 2012.

[33] L. D. Ramire, E. V. C. Marcos, D. A. S. Godoy, and F. C. de Souza-Santana, "Association of class I and II HLA alleles and haplotypes with susceptibility to vitiligo: a study of patients with vitiligo from southeast Brazil," International Journal of Dermatology, vol. 55, no. 6, pp. 347-355, 2016. 


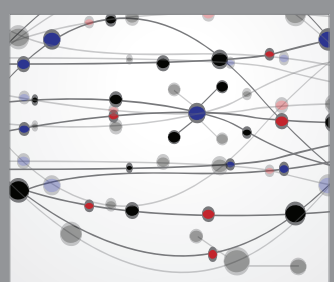

The Scientific World Journal
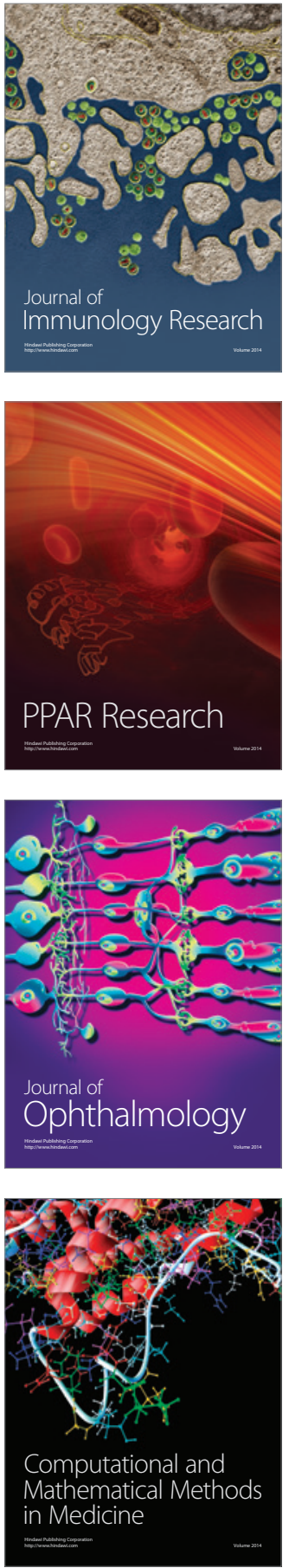

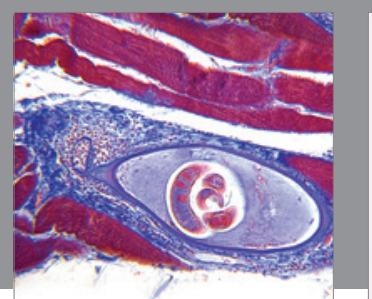

Gastroenterology Research and Practice

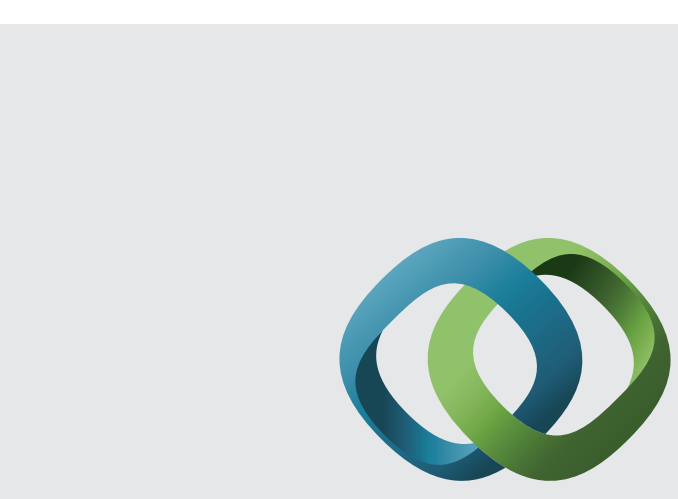

\section{Hindawi}

Submit your manuscripts at

http://www.hindawi.com
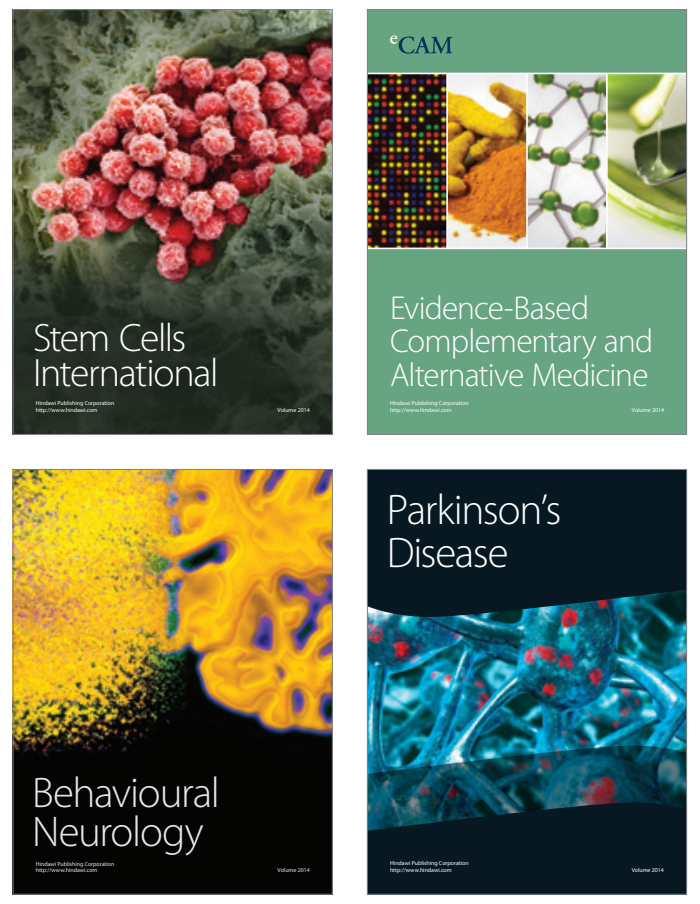
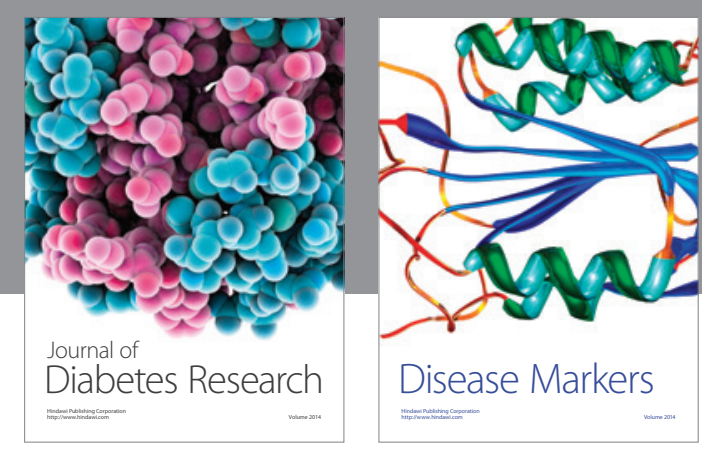

Disease Markers
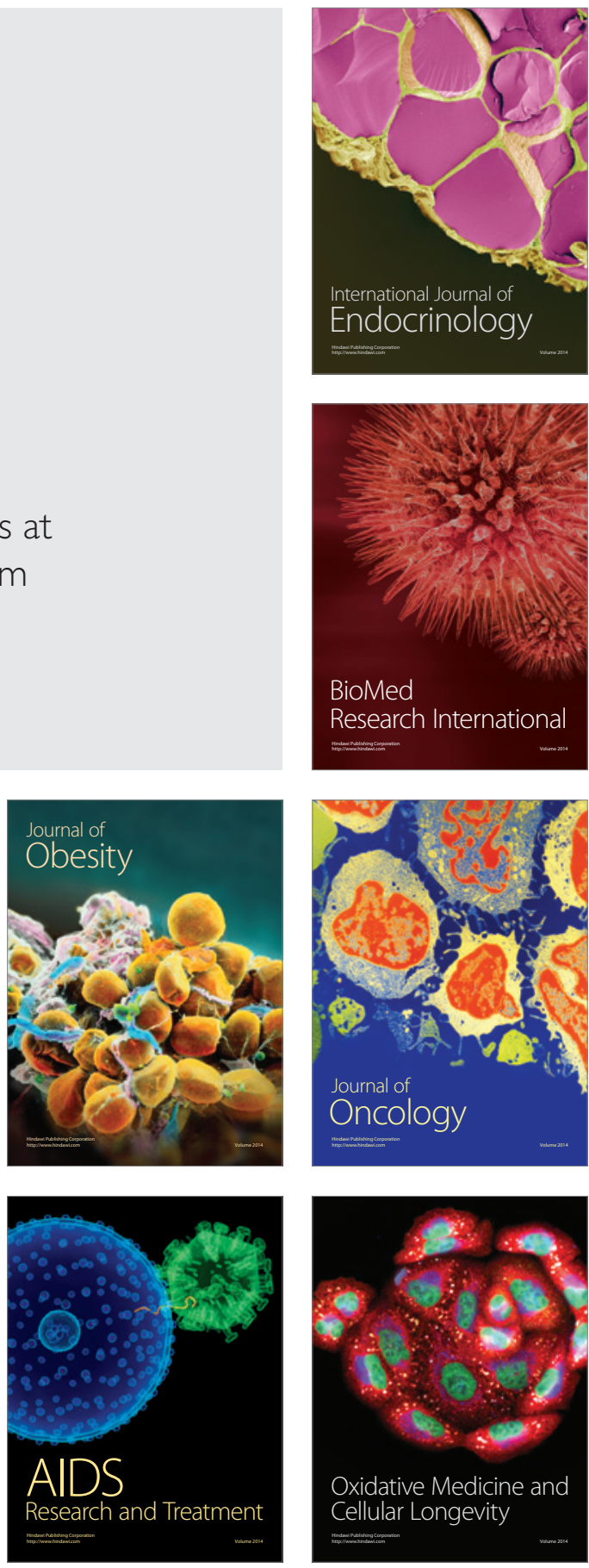